\title{
Extrinsic and intrinsic regulation of axon regeneration at a crossroads
}

\author{
Andrew Kaplan, Stephan Ong Tone and Alyson E. Fournier* \\ Department of Neurology/Neurosurgery, Montréal Neurological Institute, McGill University, Montréal, QC, Canada
}

Repair of the injured spinal cord is a major challenge in medicine. The limited intrinsic regenerative response mounted by adult central nervous system (CNS) neurons is further hampered by astrogliosis, myelin debris and scar tissue that characterize the damaged CNS. Improved axon regeneration and recovery can be elicited by targeting extrinsic factors as well as by boosting neuron-intrinsic growth regulators. Our knowledge of the molecular basis of intrinsic and extrinsic regulators of regeneration has expanded rapidly, resulting in promising new targets to promote repair. Intriguingly certain neuron-intrinsic growth regulators are emerging as promising targets to both stimulate growth and relieve extrinsic inhibition of regeneration. This crossroads between the intrinsic and extrinsic aspects of spinal cord injury is a promising target for effective therapies for this unmet need.

\section{OPEN ACCESS}

Edited by:

Sari Hannila,

University of Manitoba, Canada

Reviewed by:

Lars Klimaschewski, Medical University of Innsbruck,

Austria

William Ben Cafferty,

Yale University, USA

${ }^{*}$ Correspondence:

Alyson E. Fournier,

Department of

Neurology/Neurosurgery, Montréal

Neurological Institute, McGill

University, 3801 University, BT-109 Montréal, QC H3A2B4 Canada

alyson.fournier@mcgill.ca

Received: 24 April 2015 Accepted: 03 June 2015 Published: 16 June 2015

Citation:

Kaplan A, Ong Tone S and Fournier $A E$ (2015) Extrinsic and intrinsic regulation of axon regeneration at a

crossroads.

Front. Mol. Neurosci. 8:27. doi: 10.3389/fnmol.2015.00027

\section{Keywords: spinal cord injury, CNS regeneration, PNS, conditioning lesion, RhoA, cAMP}

\section{Introduction}

The adult mammalian central nervous system (CNS) has a poor ability to regenerate and restore function after injury. The pioneering work of Aguayo and colleagues showing that CNS neurons can grow into peripheral nerve grafts, but stop when they re-encounter the CNS led to the predominance of the hypothesis that the failure of axons to regenerate is attributed to the presence of inhibitory factors in the CNS microenvironment (David and Aguayo, 1981; Yiu and He, 2006). Indeed, it is now recognized that CNS myelin contains myelin-associated inhibitors (MAIs) including Nogo, myelin-associated glycoprotein (MAG) and oligodendrocyte myelin glycoprotein (OMgp) that collapse axonal growth cones and inhibit growth. The deposition of chondroitin sulfate proteoglycans (CSPGs) by reactive astrocytes also presents a formidable barrier to axon regeneration through sites of injury (Yiu and He, 2006). Targeting these extrinsic inhibitory factors has led to modest improvements in axonal plasticity and functional recovery after CNS injury. Important work spearheaded by Marie Filbin and colleagues revealed a critical role for the intrinsic state of CNS neurons in mediating extrinsic inhibition of axon regeneration. Filbin and colleagues demonstrated that an age-associated decline in neuronal cAMP underlies a gain of sensitivity to myelin-mediated inhibition (Cai et al., 2001). The notion that the neuron-intrinsic growth state can regulate the sensitivity of the injured axon to extrinsic factors is also supported by the enhanced axon regeneration in the spinal cord that can be elicited by a preconditioning lesion of the peripheral nerve processes (Mar et al., 2014a). More recently, it has been shown that stimulating intrinsic growth potential by neuronal knockout of negative regulators of growth, including phosphatase and tensin homolog (PTEN) and suppressor of cytokine signaling 3 (SOCS3), can induce striking long-distance axon regeneration after CNS injury (Liu et al., 2010; Sun et al., 2011). Furthermore, engraftment of neural stem cells into transected rodent spinal cords can result in impressive long-distance growth of grafted cells (Lu et al., 2012). These studies provide proof-of-concept that robust neuron-intrinsic growth potential is able to overcome 
the inhibitory nature of the injured CNS. Here we will review some novel therapeutic strategies that have been developed based on our expanding knowledge of the molecular basis of intrinsic axon growth and extrinsic outgrowth inhibition (Figure 1). We will also highlight several recent examples of intrinsic regulators that dually affect the neuronal response to the inhibitory CNS environment. These findings have blurred the distinction between an extrinsic or an intrinsic origin of CNS regeneration failure and support the idea that a dynamic interplay between the two determines whether an axon regenerates. A better understanding of this crossroads between extrinsic and intrinsic regulation of axon regeneration will contribute to the conception of therapies to stimulate CNS repair.

\section{Extrinsic Influences on Neural Repair}

\section{Myelin-associated Inhibitors}

The identification of the inhibitory ligands and neuronal receptors that mediate myelin-dependent inhibition of regeneration has facilitated the development of selective antagonists to neutralize the inhibitory effect of myelin proteins. MAIs in CNS myelin bind to either paired immunoglobulin repeat B (PirB; Atwal et al., 2008) or Nogo receptor (NgR), which forms a complex with LINGO-1 (LRR and Ig containing Nogo Receptor interacting protein) and p75NTR (p75 neurotrophin receptor) or TROY (tumor necrosis factor receptor superfamily member 19; Yiu and He, 2006). One strategy to target these interactions has been to generate peptide mimics of ligands to compete for receptor binding. An early example of this is the Nogo extracellular peptide (NEP1-40), a peptide derived from the Nogo-66 NgR-binding region of Nogo to compete with the MAIs for $\mathrm{NgR}$ binding (Grandpré et al., 2002). NEP1-40 was shown to abrogate Nogo-dependent neurite outgrowth inhibition and promote corticospinal tract regeneration after dorsal hemisection SCI (Grandpré et al., 2002; Li and Strittmatter, 2003), but a later replication study did not observe significant effects on axon regeneration (Steward et al., 2008). Vaccines (Huang et al., 1999) and monoclonal antibodies (Zörner and Schwab, 2010) have also been developed to neutralize the MAIs, with reported improvements on regeneration and functional recovery. However, deletion of $\mathrm{NgR}$ or Nogo, MAG and OMgp in mice has had more variable effects on regeneration and recovery (Zheng et al., 2003, 2005; Lee et al., 2009, 2010; Cafferty et al., 2010). While deletion of Nogo or $\mathrm{NgR}$ has negligible to modest effects on axon regeneration after dorsal hemisection SCI, a unilateral pyramidotomy injury to the CST results in an impressive collateral sprouting response of uninjured axons into the denervated side of the spinal cord (Cafferty and Strittmatter, 2006). Furthermore, a soluble NgR decoy receptor, $\mathrm{NgR}(310)$ ecto-Fc that neutralizes Nogo, MAG, and OMgp shows promise in promoting axonal sprouting and functional recovery in acute and chronic SCI in rodents (Ji et al., 2005; Li et al., 2005; Wang et al., 2011).

\section{The Glial Scar}

The astrocytic response to CNS injury is characterized by the production of CSPGs, which present a potent barrier to axon regeneration (Yiu and $\mathrm{He}, 2006$ ). The inhibitory action of CSPGs has been attributed to the abundance of negatively charged glycosaminoglycan (GAG) chains that decorate the protein core, which are thought to act as a poor substrate and electrostatically repel growth cones. Protein tyrosine phosphatase sigma (PTPsigma), leukocyte common antigen related phosphatase (LAR) and NgR have been identified as neuronal receptors that functionally interact with and mediate CSPG-dependent inhibition of neuron growth (Shen et al., 2009; Fisher et al., 2011; Dickendesher et al., 2012). Chondroitinase $\mathrm{ABC}$ (chABC), a bacterial enzyme that digests the GAG chains, abrogates CSPG-dependent neurite outgrowth inhibition in vitro and improves neurite sprouting and functional recovery after SCI (Bradbury et al., 2002). The efficacy of chABC alone and in combination with other approaches in promoting recovery after SCI has been independently confirmed in multiple laboratories and is a promising approach for future translation (Alilain et al., 2011; García-Alías et al., 2011; Lee et al., 2012). Mechanistically, contrary to the view that CSPGs repel growth cones, recent studies from the Silver group suggest that CSPGs in fact tightly adhere to and confine growth cones (Filous et al., 2014; Lang et al., 2015). After SCI, axons closely associate with NG2, a transmembrane CSPG expressed by a subset of oligodendrocyte progenitor cells. The presence of synaptic markers at these sites of apposition suggests the formation of stable "synapse-like" contacts between neurons and NG2+ cells (Filous et al., 2014). Furthermore, DRG neurons preferentially adhere to CSPG substrates in in vitro stripe assays. Silver and colleagues propose a model whereby CSPGs function to capture and arrest regenerating growth cones after injury instead of repelling them (Filous et al., 2014). Perhaps unexpectedly, deletion of NG2 in mice did not enhance axon regeneration after dorsal column crush, but rather resulted in more pronounced axonal dieback away from the injury site. This was attributed to the loss of NG2-mediated capture of axons after injury.

Pharmacological targeting of CSPG receptors is a strategy to relieve CSPG-dependent capture of growth cones that has shown potential in recent studies. Systemic administration of cell-penetrating peptide mimetics of the extracellular and intracellular regions of LAR by subcutaneous injection have been shown to enhance serotonergic axon sprouting and functional recovery after SCI in mice (Fisher et al., 2011). A recent report from the Silver group demonstrates that PTPsigma can be targeted in a similar fashion with a cell-permeable peptide, termed intracellular sigma peptide (ISP), to enhance functional recovery after contusive spinal cord injury in rats (Lang et al., 2015). ISP was designed to bind to the cytoplasmic wedge domain of PTPsigma. Using a CSPG gradient crossing assay, Silver and colleagues provide evidence that PTPsigma-dependent interactions with CSPGs maintain growth cones in a dystrophic state and that treatment with ISP allows for growth cone release. Based on the proposed mechanism that the interaction between PTPsigma and CSPGs acts as a "molecular velcro", it is surprizing that ISP is sufficient to overcome growth cone immobilization because ISP targets the cytoplasmic domain of PTPsigma, presumably sparing the physical interaction between 


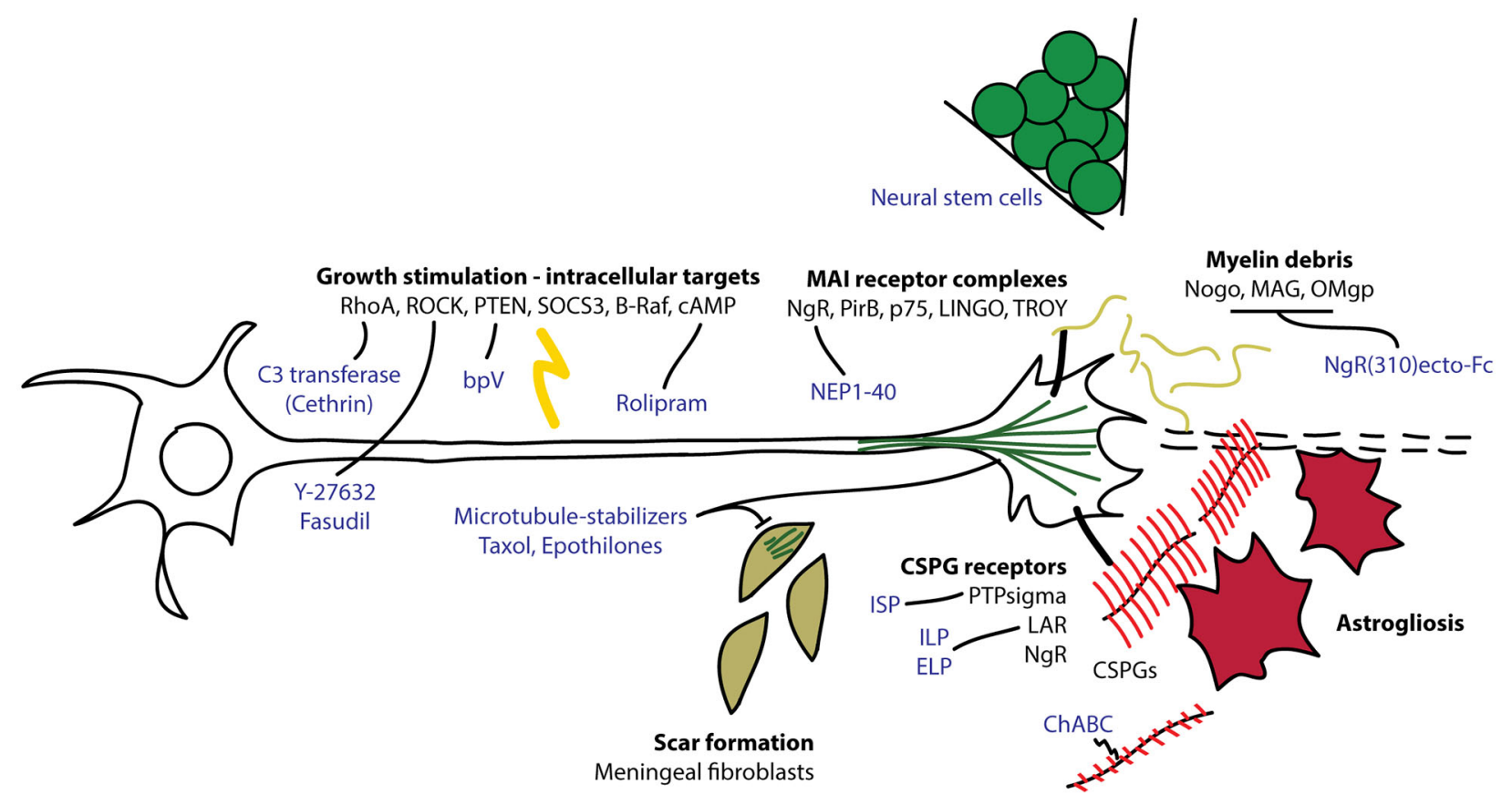

FIGURE 1 | Overview of intrinsic and extrinsic molecules involved in axon regeneration after CNS injury. Myelin debris from damaged oligodendrocytes and chondroitin sulfate proteoglycans (CSPGs) produced by reactive astrocytes interact with receptors on the growth cones to interfere with axon extension. Intracellular signaling molecules regulate the ability of the neuron to regenerate its damaged axon through the lesion environment. C3 transferase (Cethrin) inhibits RhoA to promote axon extension and cell survival. Fasudil and Y-27632 inhibit Rho kinase (ROCK) to promote axon extension. Bisperoxovanadium (bpV) inhibits phosphatase and tensin homolog (PTEN) to promote neuroprotection. Rolipram inhibits phosphodiesterase 4 (PDE4) to stabilize cAMP and promote axon extension. Microtubule stabilizers, taxol and epothilones, reduce fibrotic scarring and promote axon extension. Chondroitinase ABC (ChABC) digests CSPG glycosaminoglycan (GAG) chains (in red), relieving CSPG-dependent growth inhibition. Intracellular sigma peptide (ISP) inhibits protein tyrosine phosphatase sigma (PTPsigma) and intracellular/extracellular leukocyte common antigen related phosphatase (LAR) peptides (ILP/ELP) inhibit leukocyte antigen-related receptor (LAR) to neutralize the inhibitory effect of CSPGs and promote axon sprouting. NgR(310)ecto-Fc competes for binding to Nogo, myelin-associated glycoprotein (MAG) and Oligodendrocyte myelin glycoprotein (OMgp) to relieve myelin-dependent growth inhibition. Nogo extracellular peptide 1-40 (NEP1-40) competes for $\mathrm{NgR}$ binding to neutralize inhibitory signaling through $\mathrm{NgR}$.
CSPGs and the receptor. The relative contribution of the receptor-ligand interaction and downstream signaling events to growth cone immobilization remain unclear. Daily subcutaneous injection of ISP following spinal cord contusion in rats resulted in delayed beneficial outcomes, particularly in resumption of bladder function (Lang et al., 2015). While these studies have identified CSPG receptors as potential targets, future work could focus on developing small molecule inhibitors to attain better CNS penetrance and distribution for earlier and more efficient targeting after injury. The idea that CSPGs are critical for growth cone arrest suggests that these approaches may also be efficacious in chronic SCI.

\section{Intrinsic Influences on Neural Repair}

\section{The Conditioning Lesion}

Injury to axons in the PNS is followed by neuronal expression of regeneration-associated genes (RAGs), a response that is quite limited in CNS neurons (Mar et al., 2014a). It has therefore been hypothesized that a failure to initiate a growth program contributes to unsuccessful regeneration in the CNS. The positive effect of a peripheral conditioning lesion on CNS regeneration has been attributed to the engagement of a RAG expression response that stimulates growth (Blesch et al., 2012). One of these RAGs, c-Jun, has been shown to be critical for axon regeneration, as its deletion impairs axon regeneration and results in exacerbated cell death after peripheral nerve injury in mice (Raivich et al., 2004). The conditioning lesion has also recently been shown to enhance the axonal transport of a multitude of signaling and cytoskeleton regulators into the central process (Mar et al., 2014b). Among these include members of the 14-3-3 adaptor proteins, which have important roles in neuroprotection, axon guidance and cell growth (Shimada et al., 2013; Kaplan et al., 2014). Elevation of cAMP is also a critical component of the conditioning lesion effect and injection of cAMP into dorsal root ganglia can mimic the pro-regeneration effect of a conditioning lesion (Qiu et al., 2002). Interestingly, cAMP also regulates the sensitivity of neurons to MAIs and CSPGs (Cai et al., 2001). The Filbin group demonstrated that an age-associated decline of neuronal cAMP correlates with an acquisition of responsiveness to myelin-mediated inhibition of growth and that pharmacological elevation of cAMP renders adult neurons insensitive to myelin and CSPGs (Cai et al., 2001). This reinforces the concept that extrinsic inhibitors rely 
on the intrinsic sensitivity of the neuron in order to inhibit growth and suggests that targeting neuron-intrinsic signaling can relieve the influence of the inhibitory microenvironment. Supporting a direct link between cAMP and responsiveness to myelin, a recent study shows that enhanced cAMP and protein kinase A (PKA) signaling stimulates the proteolysis of Nogo through activation of the ubiquitin ligase praja2 (Sepe et al., 2014). cAMP is a promising target for development and rolipram, a phosphodiesterase 4 (PDE4) inhibitor that elevates intracellular cAMP, has been shown to enhance regeneration and recovery after SCI in rodents (Lu et al., 2004; Nikulina et al., 2004; Pearse et al., 2004; Kadoya et al., 2009; Costa et al., 2013).

\section{MAI Signaling}

The actin-regulating small GTPase RhoA and downstream effector Rho kinase (ROCK) are extensively studied mediators of neurite outgrowth inhibition in the CNS. MAIs have been shown to increase the levels of active GTP-bound RhoA (Dubreuil et al., 2003) and one study suggests that the dual Rac and Rho guanine exchange factor (GEF), Kalirin9, functionally interacts with p75 in complex with $\mathrm{NgR} 1$ to stimulate RhoA activation in response to receptor activation (Harrington et al., 2008). Nogo also stimulates the phosphorylation and membrane translocation of ROCK (Alabed et al., 2006). Inhibition of ROCK stimulates neurite outgrowth on myelin and infusion of the small molecule ROCK inhibitors Y-27632 or fasudil into the injured rat spinal cord improves locomotor recovery (Fournier et al., 2003; Sung et al., 2003). However, while ROCK inhibition enhances neurite outgrowth on myelin, a persistent myelin-induced reduction of growth relative to enhanced baseline growth on permissive substrates is often reported (Fournier et al., 2003; Alabed et al., 2006; Ahmed et al., 2009), suggesting that ROCK inhibition fails to fully de-sensitize neurons to myelin-dependent growth inhibition. It is therefore difficult to attribute the positive effects of ROCK inhibition in SCI to a reduction in the sensitivity of the axons to myelin or simply to an intrinsic stimulation of outgrowth. Our group has identified a Nogo-induced interaction between RhoA and collapsin response mediator protein 4 (CRMP4) as a functional signaling event that can be targeted with a competitive peptide antagonist called C4RIP to block myelin-induced neurite outgrowth inhibition (Alabed et al., 2007). Interestingly, C4RIP does not enhance baseline neurite outgrowth (Alabed et al., 2007), suggesting the specific involvement of a CRMP4-RhoA complex engaged by MAIs. RhoA can be specifically inhibited with the Clostridium botulinum exoenzyme C3 transferase, which ADP-ribosylates and inactivates RhoA. Treatment of animals with C3 improves locomotor recovery in contusion and dorsal hemisection injury models (Boato et al., 2010). C3 has a dual effect of enhancing neurite outgrowth in the presence of myelin (Dergham et al., 2002) and promoting cell survival after SCI (Dubreuil et al., 2003). Interestingly, C3 has also been shown to stimulate neurite outgrowth and induce Erk and Akt phosphorylation independent of its ability to inhibit RhoA, suggesting that its mechanism-of-action has yet to be fully elucidated (Auer et al., 2012). Detrimental effects of C3
(Fournier et al., 2003; Sung et al., 2003) observed in other studies are potentially due to endotoxin contamination and lack of a cell-penetrating peptide (McKerracher and Higuchi, 2006). The positive outcomes with $\mathrm{C} 3$ have translated into a phase I/II clinical trial with Cethrin (BA-210), a cell-permeable form of C3 that is delivered locally over the dura mater in a fibrin sealant during spinal surgery. Results from the Cethrin trial have shown promising improvements of locomotor function compared to historical statistics (McKerracher and Anderson, 2013).

\section{Growth Stimulation}

Recent studies have provided a strong rationale for targeting intracellular growth regulators as a means to both drive neuron growth and relieve sensitivity to extrinsic inhibition of growth. Initial studies have explored strategies to stimulate intrinsic growth potential by genetically manipulating the expression of master regulators of cellular growth that are considered classical tumor suppressors or oncogenes. PTEN is a tumor suppressor that antagonizes the PI3K-Akt-mTOR pathway (Song et al., 2012). The He lab reported an ageassociated down-regulation of mTOR activity in adult cortical neurons and showed that conditional knockout of PTEN and consequent increase in mTOR activity in mouse cortex promotes regeneration of corticospinal axons after dorsal hemisection injury (Liu et al., 2010). Another study showed that combined deletion of PTEN and SOCS3, a negative regulator of the JAK/STAT pathway, synergize to promote axon regeneration in injured optic nerve (Sun et al., 2011). These studies were noted for extraordinarily extensive longdistance axon regeneration that had not been observed with any prior intervention. These positive outcomes have resulted in a greater focus on manipulation of intrinsic regulation of neuron growth as an approach for drug development. Chronic targeting of tumor suppressors poses a concern for oncogenesis, but an acute regimen may be an effective strategy to initiate a regenerative response after injury. One study found that systemic administration of the PTEN inhibitor bisperoxovanadium (bpVpic) twice daily for 1 week improved forelimb motor function and was neuroprotective (Walker et al., 2012).

A complementary strategy is to enhance the activity or expression of growth-promoting molecules. A recent study showed that expression of a point-mutated kinase activated version of the B-Raf oncogene (V600E B-Raf) results in robust regeneration of retinal axons after optic nerve injury and regeneration of dorsal root ganglion neurons into the dorsal root entry zone after crush injury (O'Donovan et al., 2014). Further enhancement of regeneration was observed with combined knockout of PTEN. How these manipulations influence neuronal responses to extrinsic inhibition are poorly understood. It was recently shown that codeletion of Nogo and PTEN does not cause more corticospinal axon sprouting after unilateral pyramidotomy, but results in improved longdistance axon regeneration after dorsal hemisection, compared to PTEN deletion alone (Geoffroy et al., 2015). The lack of a combinatorial effect of PTEN and Nogo deletion on 
sprouting suggests that perhaps PTEN deletion affords maximal de-sensitization to Nogo. One study provides evidence that PTEN knockout neurons are less sensitive to MAG-dependent neurite outgrowth inhibition (Perdigoto et al., 2011), while another study has shown that Erk, a downstream effector of Raf, can also relieve MAG-dependent inhibition (Gao et al., 2003). It remains unclear whether these pathways are directly involved in MAI signaling or whether they act in parallel to alter MAI signal-transduction efficiency. Interestingly, a recent study shows that myelin and CSPGs stimulate the expression of pro-growth immediate early genes through serum response factor (SRF) and Erk activation. This may function as a protective response because expression of constitutively active SRF was shown to overcome myelin and CSPG-dependent neurite outgrowth inhibition (Stern and Knoll, 2014).

\section{Neural Stem Cells}

The ability of implanted neural stem cells to survive and functionally integrate into injured host spinal cord in rodents also suggests that the inhibitory nature of the injured CNS can be overcome by neurons with vigorous growth capacity (Lu et al., 2012, 2014). In studies from the Tuszynski group, implanted neural stem cells were shown to extend long axons throughout the grey and white matter of transected host spinal cords, establishing an electrophysiological bridge across the injury. A recently published study confirmed the ability of grafted neural stem cells to integrate into host spinal cords, but also noted the presence of ectopic colonies of donor cells throughout the spinal cord and brain stem in half of the animals (Steward et al., 2014). This highlights the caution that must be exercised in the development of neural stem cell therapies, as implanted cells can give rise to tumors and exuberant synaptic connections could result in unfavorable behavioral and sensory side effects including neuropathic pain (Hofstetter et al., 2005). Nonetheless, these studies also serve as proof-of-principle that neurons with high growth capacity are capable of extensive growth in the injured CNS despite the presence of inhibitory factors.

\section{References}

Ahmed, Z., Berry, M., and Logan, A. (2009). ROCK inhibition promotes adult retinal ganglion cell neurite outgrowth only in the presence of growth promoting factors. Mol. Cell. Neurosci. 42, 128-133. doi: 10.1016/j.mcn.2009. 06.005

Alabed, Y. Z., Grados-Munro, E., Ferraro, G. B., Hsieh, S. H., and Fournier, A. E. (2006). Neuronal responses to myelin are mediated by rho kinase. J. Neurochem. 96, 1616-1625. doi: 10.1111/j.1471-4159.2006. 03670.x

Alabed, Y. Z., Pool, M., Ong Tone, S., and Fournier, A. E. (2007). Identification of CRMP4 as a convergent regulator of axon outgrowth inhibition. J. Neurosci. 27, 1702-1711. doi: 10.1523/jneurosci.5055-06.2007

Alilain, W. J., Horn, K. P., Hu, H., Dick, T. E., and Silver, J. (2011). Functional regeneration of respiratory pathways after spinal cord injury. Nature 475, 196-200. doi: 10.1038/nature10199

Atwal, J. K., Pinkston-Gosse, J., Syken, J., Stawicki, S., Wu, Y., Shatz, C., et al. (2008). PirB is a functional receptor for myelin inhibitors of axonal regeneration. Science 322, 967-970. doi: 10.1126/science.1161151

\section{Conclusion}

Both the extrinsic and intrinsic aspects of the injury response together forge the basis for regeneration failure. Peripheral conditioning lesions combined with chABC and $\mathrm{NgR}(310)$ ecto have been shown to provide superior benefit to single interventions, supporting the idea that simultaneously stimulating neuron-intrinsic growth potential and neutralizing extrinsic inhibition can provide maximal efficacy in promoting neural repair (Wang et al., 2012). SCI therapies that take both of these contributors into consideration can come in the form of drug combinations or single multi-action drugs. The use of multi-action drugs is an attractive strategy to simultaneously manipulate several pathophysiological features of the injury response. For example, work from the Bradke group has shown that local administration of low dose taxol, a potent microtubule-stabilizing drug, exerts manifold beneficial effects by reducing CSPG production and fibrosis, inhibiting meningeal fibroblast migration and stimulating axon extension after SCI (Hellal et al., 2011). A recent report from the Bradke group shows that systemic administration of another microtubule stabilizer, epothilone B, similarly inhibits scarring and promotes axon regeneration (Ruschel et al., 2015). Both drugs are approved chemotherapeutics, however epothilone $\mathrm{B}$, but not taxol, is CNS-penetrant. CNS-penetrant multiaction pharmaceutical agents that can be given systemically hold the most promise for translation into practical and effective treatments for SCI. The repurposing of approved drugs for indication in SCI is an attractive approach to achieve accelerated approval for this serious unmet need. However, the identification of new drugs that have multiple activities, including modulation of astrogliosis, neuroprotection and neurite outgrowth induction, may offer superior efficacy in stimulating CNS repair, but will also pose challenges for safety and tolerability.

\section{Author Contributions}

AK, SOT and AEF wrote the manuscript.

Auer, M., Schweigreiter, R., Hausott, B., Thongrong, S., Höltje, M., Just, I., et al. (2012). Rho-independent stimulation of axon outgrowth and activation of the ERK and Akt signaling pathways by $\mathrm{C} 3$ transferase in sensory neurons. Front. Cell. Neurosci. 6:43. doi: 10.3389/fncel.2012.00043

Blesch, A., Lu, P., Tsukada, S., Alto, L. T., Roet, K., Coppola, G., et al. (2012). Conditioning lesions before or after spinal cord injury recruit broad genetic mechanisms that sustain axonal regeneration: superiority to camp-mediated effects. Exp. Neurol. 235, 162-173. doi: 10.1016/j.expneurol.2011.12.037

Boato, F., Hendrix, S., Huelsenbeck, S. C., Hofmann, F., Grosse, G., Djalali, S., et al. (2010). C3 peptide enhances recovery from spinal cord injury by improved regenerative growth of descending fiber tracts. J. Cell Sci. 123, 1652-1662. doi: $10.1242 /$ jcs.066050

Bradbury, E. J., Moon, L. D., Popat, R. J., King, V. R., Bennett, G. S., Patel, P. N., et al. (2002). Chondroitinase $\mathrm{ABC}$ promotes functional recovery after spinal cord injury. Nature 416, 636-640. doi: 10.1038/416636a

Cafferty, W. B., Duffy, P., Huebner, E., and Strittmatter, S. M. (2010). MAG and OMgp synergize with Nogo-A to restrict axonal growth and neurological recovery after spinal cord trauma. J. Neurosci. 30, 6825-6837. doi: 10. 1523/jneurosci.6239-09.2010 
Cafferty, W. B., and Strittmatter, S. M. (2006). The Nogo-Nogo receptor pathway limits a spectrum of adult CNS axonal growth. J. Neurosci. 26, 12242-12250. doi: 10.1523/jneurosci.3827-06.2006

Cai, D., Qiu, J., Cao, Z., Mcatee, M., Bregman, B. S., and Filbin, M. T. (2001). Neuronal cyclic AMP controls the developmental loss in ability of axons to regenerate. J. Neurosci. 21, 4731-4739.

Costa, L. M., Pereira, J. E., Filipe, V. M., Magalhães, L. G., Couto, P. A., GonzaloOrden, J. M., et al. (2013). Rolipram promotes functional recovery after contusive thoracic spinal cord injury in rats. Behav. Brain Res. 243, 66-73. doi: 10.1016/j.bbr.2012.12.056

David, S., and Aguayo, A. J. (1981). Axonal elongation into peripheral nervous system "bridges" after central nervous system injury in adult rats. Science 214, 931-933. doi: 10.1126/science.6171034

Dergham, P., Ellezam, B., Essagian, C., Avedissian, H., Lubell, W. D., and Mckerracher, L. (2002). Rho signaling pathway targeted to promote spinal cord repair. J. Neurosci. 22, 6570-6577.

Dickendesher, T. L., Baldwin, K. T., Mironova, Y. A., Koriyama, Y., Raiker, S. J., Askew, K. L., et al. (2012). NgR1 and NgR3 are receptors for chondroitin sulfate proteoglycans. Nat. Neurosci. 15, 703-712. doi: 10.1038/nn.3070

Dubreuil, C. I., Winton, M. J., and Mckerracher, L. (2003). Rho activation patterns after spinal cord injury and the role of activated Rho in apoptosis in the central nervous system. J. Cell Biol. 162, 233-243. doi: 10.1083/jcb. 200301080

Filous, A. R., Tran, A., Howell, C. J., Busch, S. A., Evans, T. A., Stallcup, W. B., et al. (2014). Entrapment via synaptic-like connections between NG2 proteoglycan+ cells and dystrophic axons in the lesion plays a role in regeneration failure after spinal cord injury. J. Neurosci. 34, 16369-16384. doi: 10.1523/jneurosci.130914.2014

Fisher, D., Xing, B., Dill, J., Li, H., Hoang, H. H., Zhao, Z., et al. (2011). Leukocyte common antigen-related phosphatase is a functional receptor for chondroitin sulfate proteoglycan axon growth inhibitors. J. Neurosci. 31, 14051-14066. doi: 10.1523/jneurosci.1737-11.2011

Fournier, A. E., Takizawa, B. T., and Strittmatter, S. M. (2003). Rho kinase inhibition enhances axonal regeneration in the injured CNS. J. Neurosci. 23, 1416-1423.

Gao, Y., Nikulina, E., Mellado, W., and Filbin, M. T. (2003). Neurotrophins elevate cAMP to reach a threshold required to overcome inhibition by MAG through extracellular signal-regulated kinase-dependent inhibition of phosphodiesterase. J. Neurosci. 23, 11770-11777.

García-Alías, G., Petrosyan, H. A., Schnell, L., Horner, P. J., Bowers, W. J., Mendell, L. M., et al. (2011). Chondroitinase ABC combined with neurotrophin NT-3 secretion and NR2D expression promotes axonal plasticity and functional recovery in rats with lateral hemisection of the spinal cord. J. Neurosci. 31, 17788-17799. doi: 10.1523/jneurosci.4308-11.2011

Geoffroy, C. G., Lorenzana, A. O., Kwan, J. P., Lin, K., Ghassemi, O., Ma, A., et al. (2015). Effects of PTEN and Nogo codeletion on corticospinal axon sprouting and regeneration in mice. J. Neurosci. 35, 6413-6428. doi: 10.1523/jneurosci. 4013-14.2015

Grandpré, T., Li, S., and Strittmatter, S. M. (2002). Nogo-66 receptor antagonist peptide promotes axonal regeneration. Nature 417, 547-551. doi: 10. $1038 / 417547 \mathrm{a}$

Harrington, A. W., Li, Q. M., Tep, C., Park, J. B., He, Z., and Yoon, S. O. (2008). The role of Kalirin9 in p75/nogo receptor-mediated RhoA activation in cerebellar granule neurons. J. Biol. Chem. 283, 24690-24697. doi: 10.1074/jbc. m802188200

Hellal, F., Hurtado, A., Ruschel, J., Flynn, K. C., Laskowski, C. J., Umlauf, M., et al. (2011). Microtubule stabilization reduces scarring and causes axon regeneration after spinal cord injury. Science 331, 928-931. doi: 10. 1126/science. 1201148

Hofstetter, C. P., Holmström, N. A., Lilja, J. A., Schweinhardt, P., Hao, J., Spenger, C., et al. (2005). Allodynia limits the usefulness of intraspinal neural stem cell grafts; directed differentiation improves outcome. Nat. Neurosci. 8, 346-353. doi: $10.1038 / \mathrm{nn} 1405$

Huang, D. W., Mckerracher, L., Braun, P. E., and David, S. (1999). A therapeutic vaccine approach to stimulate axon regeneration in the adult mammalian spinal cord. Neuron 24, 639-647. doi: 10.1016/s0896-6273(00)81118-6

Ji, B., Li, M., Budel, S., Pepinsky, R. B., Walus, L., Engber, T. M., et al. (2005). Effect of combined treatment with methylprednisolone and soluble Nogo-66 receptor after rat spinal cord injury. Eur. J. Neurosci. 22, 587-594. doi: 10.1111/j.14609568.2005.04241.x

Kadoya, K., Tsukada, S., Lu, P., Coppola, G., Geschwind, D., Filbin, M. T., et al. (2009). Combined intrinsic and extrinsic neuronal mechanisms facilitate bridging axonal regeneration one year after spinal cord injury. Neuron 64, 165-172. doi: 10.1016/j.neuron.2009.09.016

Kaplan, A., Kent, C. B., Charron, F., and Fournier, A. E. (2014). Switching responses: spatial and temporal regulators of axon guidance. Mol. Neurobiol. 49, 1077-1086. doi: 10.1007/s12035-013-8582-8

Lang, B. T., Cregg, J. M., Depaul, M. A., Tran, A. P., Xu, K., Dyck, S. M., et al. (2015). Modulation of the proteoglycan receptor PTPsigma promotes recovery after spinal cord injury. Nature 518, 404-408. doi: 10.1038/nature13974

Lee, H. J., Bian, S., Jakovcevski, I., Wu, B., Irintchev, A., and Schachner, M. (2012). Delayed applications of $\mathrm{L} 1$ and chondroitinase ABC promote recovery after spinal cord injury. J. Neurotrauma 29, 1850-1863. doi: 10.1089/neu.2011.2290

Lee, J. K., Chan, A. F., Luu, S. M., Zhu, Y., Ho, C., Tessier-Lavigne, M., et al. (2009). Reassessment of corticospinal tract regeneration in Nogo-deficient mice. J. Neurosci. 29, 8649-8654. doi: 10.1523/JNEUROSCI.1864-09.2009

Lee, J. K., Geoffroy, C. G., Chan, A. F., Tolentino, K. E., Crawford, M. J., Leal, M. A., et al. (2010). Assessing spinal axon regeneration and sprouting in Nogo-, MAG- and OMgp-deficient mice. Neuron 66, 663-670. doi: 10.1016/j.neuron. 2010.05.002

Li, S., Kim, J. E., Budel, S., Hampton, T. G., and Strittmatter, S. M. (2005). Transgenic inhibition of Nogo-66 receptor function allows axonal sprouting and improved locomotion after spinal injury. Mol. Cell. Neurosci. 29, 26-39. doi: 10.1016/j.mcn.2004.12.008

Li, S., and Strittmatter, S. M. (2003). Delayed systemic Nogo-66 receptor antagonist promotes recovery from spinal cord injury. J. Neurosci. 23, 4219-4227.

Liu, K., Lu, Y., Lee, J. K., Samara, R., Willenberg, R., Sears-Kraxberger, I., et al. (2010). PTEN deletion enhances the regenerative ability of adult corticospinal neurons. Nat. Neurosci. 13, 1075-1081. doi: 10.1038/nn.2603

Lu, P., Wang, Y., Graham, L., Mchale, K., Gao, M., Wu, D., et al. (2012). Longdistance growth and connectivity of neural stem cells after severe spinal cord injury. Cell 150, 1264-1273. doi: 10.1016/j.cell.2012.08.020

Lu, P., Woodruff, G., Wang, Y., Graham, L., Hunt, M., Wu, D., et al. (2014). Longdistance axonal growth from human induced pluripotent stem cells after spinal cord injury. Neuron 83, 789-796. doi: 10.1016/j.neuron.2014.07.014

Lu, P., Yang, H., Jones, L. L., Filbin, M. T., and Tuszynski, M. H. (2004). Combinatorial therapy with neurotrophins and cAMP promotes axonal regeneration beyond sites of spinal cord injury. J. Neurosci. 24, 6402-6409. doi: 10.1523/jneurosci.1492-04.2004

Mar, F. M., Bonni, A., and Sousa, M. M. (2014a). Cell intrinsic control of axon regeneration. EMBO Rep. 15, 254-263. doi: 10.1002/embr.201337723

Mar, F. M., Simões, A. R., Leite, S., Morgado, M. M., Santos, T. E., Rodrigo, I. S., et al. (2014b). CNS axons globally increase axonal transport after peripheral conditioning. J. Neurosci. 34, 5965-5970. doi: 10.1523/jneurosci.4680-13.2014

McKerracher, L., and Anderson, K. D. (2013). Analysis of recruitment and outcomes in the phase I/IIa Cethrin clinical trial for acute spinal cord injury. J. Neurotrauma 30, 1795-1804. doi: 10.1089/neu.2013.2909

McKerracher, L., and Higuchi, H. (2006). Targeting Rho to stimulate repair after spinal cord injury. J. Neurotrauma 23, 309-317. doi: 10.1089/neu.2006.23.309

Nikulina, E., Tidwell, J. L., Dai, H. N., Bregman, B. S., and Filbin, M. T. (2004). The phosphodiesterase inhibitor rolipram delivered after a spinal cord lesion promotes axonal regeneration and functional recovery. Proc. Natl. Acad. Sci. U S A 101, 8786-8790. doi: 10.1073/pnas.0402595101

O’Donovan, K. J., Ma, K., Guo, H., Wang, C., Sun, F., Han, S. B., et al. (2014). B-RAF kinase drives developmental axon growth and promotes axon regeneration in the injured mature CNS. J. Exp. Med. 211, 801-814. doi: 10. 1084/jem.20131780

Pearse, D. D., Pereira, F. C., Marcillo, A. E., Bates, M. L., Berrocal, Y. A., Filbin, M. T., et al. (2004). cAMP and Schwann cells promote axonal growth and functional recovery after spinal cord injury. Nat. Med. 10, 610-616. doi: 10. 1038/nm1056

Perdigoto, A. L., Chaudhry, N., Barnes, G. N., Filbin, M. T., and Carter, B. D. (2011). A novel role for PTEN in the inhibition of neurite outgrowth by myelinassociated glycoprotein in cortical neurons. Mol. Cell. Neurosci. 46, 235-244. doi: 10.1016/j.mcn.2010.09.006 
Qiu, J., Cai, D., Dai, H., Mcatee, M., Hoffman, P. N., Bregman, B. S., et al. (2002). Spinal axon regeneration induced by elevation of cyclic AMP. Neuron 34, 895-903. doi: 10.1016/s0896-6273(02)00730-4

Raivich, G., Bohatschek, M., Da Costa, C., Iwata, O., Galiano, M., Hristova, M., et al. (2004). The AP-1 transcription factor c-Jun is required for efficient axonal regeneration. Neuron 43, 57-67. doi: 10.1016/j.neuron.2004. 06.005

Ruschel, J., Hellal, F., Flynn, K. C., Dupraz, S., Elliott, D. A., Tedeschi, A., et al. (2015). Systemic administration of epothilone B promotes axon regeneration after spinal cord injury. Science 348, 347-352. doi: 10.1126/science.aaa2958

Sepe, M., Lignitto, L., Porpora, M., Delle Donne, R., Rinaldi, L., Belgianni, G., et al. (2014). Proteolytic control of neurite outgrowth inhibitor NOGO-A by the cAMP/PKA pathway. Proc. Natl. Acad. Sci. US A 111, 15729-15734. doi: 10. 1073/pnas.1410274111

Shen, Y., Tenney, A. P., Busch, S. A., Horn, K. P., Cuascut, F. X., Liu, K., et al. (2009). PTPsigma is a receptor for chondroitin sulfate proteoglycan, an inhibitor of neural regeneration. Science 326, 592-596. doi: 10.1126/science. 1178310

Shimada, T., Fournier, A. E., and Yamagata, K. (2013). Neuroprotective function of 14-3-3 proteins in neurodegeneration. Biomed Res. Int. 2013:564534. doi: 10. $1155 / 2013 / 564534$

Song, M. S., Salmena, L., and Pandolfi, P. P. (2012). The functions and regulation of the PTEN tumour suppressor. Nat. Rev. Mol. Cell Biol. 13, 283-296. doi: 10. 1038/nrm3330

Stern, S., and Knoll, B. (2014). CNS axon regeneration inhibitors stimulate an immediate early gene response via MAP kinase-SRF signaling. Mol. Brain 7:86. doi: 10.1186/s13041-014-0086-6

Steward, O., Sharp, K. G., and Matsudaira Yee, K. (2014). Long-distance migration and colonization of transplanted neural stem cells. Cell 156, 385-387. doi: 10. 1016/j.cell.2014.01.017

Steward, O., Sharp, K., Yee, K. M., and Hofstadter, M. (2008). A re-assessment of the effects of a Nogo-66 receptor antagonist on regenerative growth of axons and locomotor recovery after spinal cord injury in mice. Exp. Neurol. 209, 446-468. doi: 10.1016/j.expneurol.2007.12.010

Sun, F., Park, K. K., Belin, S., Wang, D., Lu, T., Chen, G., et al. (2011). Sustained axon regeneration induced by co-deletion of PTEN and SOCS3. Nature 480, 372-375. doi: 10.1038/nature10594
Sung, J. K., Miao, L., Calvert, J. W., Huang, L., Louis Harkey, H., and Zhang, J. H. (2003). A possible role of RhoA/Rho-kinase in experimental spinal cord injury in rat. Brain Res. 959, 29-38. doi: 10.1016/s0006-8993(02)03717-4

Walker, C. L., Walker, M. J., Liu, N. K., Risberg, E. C., Gao, X., Chen, J., et al. (2012). Systemic bisperoxovanadium activates Akt $/ \mathrm{mTOR}$, reduces autophagy and enhances recovery following cervical spinal cord injury. PLoS One 7:e30012. doi: 10.1371/journal.pone.0030012

Wang, X., Duffy, P., McGee, A. W., Hasan, O., Gould, G., Tu, N., et al. (2011). Recovery from chronic spinal cord contusion after Nogo receptor intervention. Ann. Neurol. 70, 805-821. doi: 10.1002/ana.22527

Wang, X., Hasan, O., Arzeno, A., Benowitz, L. I., Cafferty, W. B., and Strittmatter, S. M. (2012). Axonal regeneration induced by blockade of glial inhibitors coupled with activation of intrinsic neuronal growth pathways. Exp. Neurol. 237, 55-69. doi: 10.1016/j.expneurol.2012.06.009

Yiu, G., and He, Z. (2006). Glial inhibition of CNS axon regeneration. Nat. Rev. Neurosci. 7, 617-627. doi: 10.1038/nrn1956

Zheng, B., Atwal, J., Ho, C., Case, L., He, X. L., Garcia, K. C., et al. (2005). Genetic deletion of the Nogo receptor does not reduce neurite inhibition in vitro or promote corticospinal tract regeneration in vivo. Proc. Natl. Acad. Sci. U S A 102, 1205-1210. doi: 10.1073/pnas.0409026102

Zheng, B., Ho, C., Li, S., Keirstead, H., Steward, O., and Tessier-Lavigne, M. (2003). Lack of enhanced spinal regeneration in Nogo-deficient mice. Neuron 38, 213-224. doi: 10.1016/s0896-6273(03)00225-3

Zörner, B., and Schwab, M. E. (2010). Anti-Nogo on the go: from animal models to a clinical trial. Ann. N Y Acad. Sci. 1198(Suppl. 1), E22-E34. doi: 10.1111/j. 1749-6632.2010.05566.x

Conflict of Interest Statement: The authors declare that the research was conducted in the absence of any commercial or financial relationships that could be construed as a potential conflict of interest.

Copyright (C) 2015 Kaplan, Ong Tone and Fournier. This is an open-access article distributed under the terms of the Creative Commons Attribution License (CC BY). The use, distribution and reproduction in other forums is permitted, provided the original author(s) or licensor are credited and that the original publication in this journal is cited, in accordance with accepted academic practice. No use, distribution or reproduction is permitted which does not comply with these terms. 